\title{
Inseguridad alimentaria y adherencia al tratamiento antirretroviral en personas con VIH de México
}

\author{
Food insecurity and adherence to antiretroviral \\ treatment among people with HIV in Mexico
}

Diana Pérez-Salgado ${ }^{1}$

María Sandra Compean-Dardón ${ }^{1}$

Luis Ortiz-Hernández ${ }^{1}$

${ }^{1}$ Departamento de Atención a la Salud, Universidad Autónoma Metropolitana unidad Xochimilco. Calzada del Hueso 1100, Col. Villa Quietud. 04960 Coyoacán México. lortiz@correo.xoc.uam.mx
Abstract The aims of this study are: 1) To analyze the relationship between food insecurity (FI) and adherence to antiretroviral therapy (ART) among people with HIV. 2) To evaluate the contribution of four possible mediators (lack of time due to work and family responsibilities, lack of resources to satisfy needs, psychological distress and side effects of ART) to differences in adherence according to the level of FI. A cross-sectional survey with a convenience sample $(n=557)$ of people with HIV attending two public institutions in Mexico City was conducted. Variables were assessed by a questionnaire. Logistic regression models were estimated. People with moderate FI were more likely to skip a dose $(R M=2.10)$ and reported low adherence during the last month $(R M=3.35)$. Poor adherence among people with FI was explained by the side effects of ART (14.9\%), unsatisfied needs (12.5\%), lack of time (4.8\%) and psychological distress (2\%). FI may curtail the benefits of universal access policies because it is associated with lower adherence to ART. An adjustment of health services to reduce the negative effects of FI on adherence to $A R T$ is required.

Key words HIV, AIDS, Mental health, Adherence, Food security
Resumen Los objetivos del artículo son: 1) Analizar la relación entre inseguridad alimentaria (IA) y adherencia al tratamiento antiretroviral (TAR) en personas con VIH/SIDA. 2) Evaluar la contribución de cuatro posibles mediadores (responsabilidades laborales y familiares, falta de recursos para cubrir necesidades, distrés psicológico y efectos secundarios del TAR) a dicha asociación. Estudio transversal con una muestra $(n=557)$ de personas con VIH atendidas en dos instituciones públicas de la ciudad de México. Las variables fueron indagadas mediante un cuestionario. Mediante modelos de regresión logística se evaluó si los mediadores explicaban la asociación entre IA y adherencia al TAR. Las personas con IA moderada tuvieron mayor probabilidad de omisión en el mes previo $(O R=2.10)$ y baja adherencia $(O R=$ 3.35). La baja adherencia en las personas con IA fue explicada por los efectos secundarios (14.9\%), las necesidades insatisfechas (12.5\%), las responsabilidades (4.8\%) y el distrés psicológico (2.0\%). La IA puede limitar los beneficios de las políticas de acceso universal al TAR pues puede estar asociada con menor adherencia a ésta. Se requiere adecuar los servicios de salud para reducir el efecto de la IA.

Palabras clave VIH, Salud mental, Adherencia al tratamiento, Inseguridad alimentaria 


\section{Introducción}

Entre las personas con VIH, la no-adherencia al tratamiento antirretroviral (TAR) predice la progresión al síndrome con la consecuente reducción de la calidad y esperanza de vida ${ }^{1}$. La no-adherencia lleva al desarrollo de virus resistentes a múltiples, fármacos lo cual puede redundar en incremento de los costos de atención ${ }^{2}$. Además, la supresión de la carga viral puede reducir la transmisión sexual del virus ${ }^{3}$.

La inseguridad alimentaria (IA) se define como la disponibilidad limitada o incierta de alimentos nutricionalmente adecuados e inocuos o la incapacidad para adquirir alimentos en formas socialmente aceptables. En México, la mitad de los hogares experimentan algún grado de IA: $24.9 \%$ tuvieron IA leve, $14.6 \%$ presentaron IA moderada y $10.5 \%$ tuvieron IA severa ${ }^{4}$.

La mayoría de los estudios ${ }^{5,6}$ sobre la relación entre IA y no-adherencia al TAR provienen de países de ingreso bajo (Kenia, Ruanda, Uganda, Sudáfrica y Perú) o alto (Canadá, Francia y E.E.U.U.). En países de ingreso alto los hallazgos son inconsistentes: en algunos estudios la relación se ha documentado ${ }^{7}$, pero en otros la asociación se observa en ciertos grupos (e.g. hombres heterosexuales) pero no en otros (mujeres heterosexuales y varones homosexuales $)^{8}$ o bien la relación no existe ${ }^{6}$. Las investigaciones de países de bajo ingreso son cualitativas ${ }^{9-12}$ o descriptivas $^{13}$ y se han realizado en África, mientras que la escasa evidencia epidemiológica muestra que la IA se relaciona positivamente con la no-adherencia al TAR ${ }^{14,15}$; lo cual puede deberse a los escasos recursos disponibles en esas sociedades.

Por las diferencias socioeconómicas entre continentes y regiones, es difícil saber si los hallazgos observados en países de ingreso bajo o alto son extrapolables a los de ingreso medio. Por ejemplo, mientras que en África la IA severa es la más común, en Latinoamérica es más frecuente la leve; mientras que en los países de ingreso alto la prevalencia de IA es menor. El porcentaje de hogares que en el último año no tuvieron comida en E.E.U.U., México, Perú, Sudáfrica y Ruanda fue de $11.3 \%, 18.1 \%, 31.5 \%, 33.4 \%$ y $61.1 \%$, respectivamente $^{16}$.

Los programas de acceso universal al tratamiento del VIH/SIDA son comunes en países de ingreso alto y medio, lo cual no ocurre en los de ingreso bajo (como la mayoría de países de la África subsahariana). En América Latina es limitada la evidencia de la relación entre IA y adherencia al TAR. Sólo existe un estudio realizado en
Perú ${ }^{5,14}$, que es de los pocos países de la región sin acceso universal al TAR ${ }^{17}$. Por lo anterior, se esperaría que en México (un país de ingreso medio, con menor prevalencia de IA y con acceso universal al TAR) el impacto de la IA sobre la adherencia al TAR fuera menor al observado en países de ingreso bajo.

Un aspecto poco evaluado en estudios epidemiológicos son los mecanismos por los cuales la IA puede limitar la adherencia al TAR, lo cual puede permitir identificar posibles áreas de intervención. Los mecanismos que se han planteado son: los sujetos que experimentan IA dan prioridad a la satisfacción de otras necesidades por encima de su tratamiento ${ }^{5}$, la IA puede exacerbar los efectos secundarios del $\mathrm{TAR}^{9,12}$, la IA implica mayores cargas laborales y familiares ${ }^{12,18}$ y el distrés psicológico por la IA puede reducir la adherencia $^{19-21}$.

Por lo anterior, el objetivo de este trabajo fue evaluar la relación entre IA y adherencia al TAR en personas con VIH/SIDA atendidas en instituciones públicas de la ciudad de México. Además, se evaluaron la contribución de cuatro posibles mediadores (responsabilidades laborales y familiares, insatisfacción de necesidades, distrés psicológico y efectos secundarios de medicamentos) a las diferencias en adherencia de acuerdo a la IA.

\section{Materiales y métodos}

Se realizó un estudio transversal analítico con una muestra por conveniencia $(n=557)$ de las personas seropositivas atendidas en la clínica de VIH del Hospital Regional Lic. Adolfo López Mateos del Instituto de Seguridad y Servicios Sociales de los Trabajadores del Estado (ISSSTE) y en la Clínica Especializada Condesa de la Secretaria de Salud del Distrito Federal (SS-GDF). El proyecto de investigación fue aprobado por el Consejo Divisional de Ciencias Biológicas y de la Salud de la Universidad Autónoma Metropolitana unidad Xochimilco, el cual sanciona los aspectos éticos. Se obtuvo el consentimiento informado por escrito de cada participante. El trabajo de campo se realizó de octubre de 2011 a noviembre de 2012. Los criterios de inclusión fueron: ser usuario de alguna de las dos clínicas, estar bajo TAR, ser mayor de 18 años o en el caso de los menores de edad que ya no dependieran de sus tutores ni vivieran con ellos. La aplicación de los cuestionarios fue realizada por pasantes de la Licenciatura en Enfermería previamente capacitadas en técnicas de entrevista. 
Las variables fueron indagadas mediante un cuestionario. La variable dependiente fue la adherencia al TAR y fue evaluada mediante tres preguntas que indagaban si la persona había omitido la toma de medicamentos antirretrovirales durante el mes previo, la semana y el día previos a la aplicación del cuestionario. Las opciones de respuesta a esas preguntas eran sí y no. Cuando la respuesta era sí, se preguntaba cuántas veces había omitido tomar el medicamento. A partir de las respuestas a la última pregunta se creó una nueva variable que evalúa la baja adherencia durante la semana y mes previo. Se denominó como baja adherencia cuando la persona había omitido 5\% o más de las dosis prescritas de medicamentos.

La variable independiente fue la IA que fue evaluada con tres preguntas: “ ¿por falta de dinero se ha preocupado o ha estado ansioso por que no sabía si tendría dinero para comprar comida en los siguientes días?”; “ipor falta de dinero, ha tenido que comprar comida barata aunque no sea sana?” y “por falta de dinero ha dejado de comer una o varias comidas en un día?”. Estas preguntas fueron tomadas de la Escala Latinoamericana y Caribeña de Seguridad Alimentaria (ELCSA) ${ }^{4,22}$. Para cada pregunta se reagruparon las cinco opciones de respuesta formando sólo dos grupos: 1) No (respuestas "nunca” y "casi nunca”) y 2) Sí ("algunas veces", "muchas veces", "casi siempre” y "siempre"). Las tres preguntas fueron incluidas en un análisis factorial y solo emergió un factor (eigenvalue $=2.55$ ), el cual explicó el $85.0 \%$ de la varianza. El alfa de Cronbach fue de 0.92 , lo cual muestra su validez interna. Posteriormente se sumaron las respuestas afirmativas y se formaron tres grupos: 1) seguridad alimentaria (SA): ninguna respuesta afirmativa; 2) IA leve: 1 o 2 respuestas afirmativas y 3 ) IA moderada: 3 respuestas afirmativas.

Las variables mediadoras consideradas para este trabajo fueron: falta de tiempo por actividades laborales y familiares, falta de recursos para cubrir sus necesidades, distrés psicológico y efectos secundarios agudos de los medicamentos. Para evaluar la primera se consideraron las siguientes preguntas: “¿Sus obligaciones o responsabilidades con su familia hicieron que no tuviera suficiente tiempo para usted?” y “¿Sus obligaciones o responsabilidades en su trabajo hicieron que no tuviera suficiente tiempo para usted?". Las opciones de respuestas fueron iguales que para las preguntas de IA. Se sumaron las respuestas afirmativas y se consideró que tenían falta de tiempo a quienes contestaron afirmativamente a una o ambas preguntas. El alfa de Cron- bach de este índice fue 0.73 . La segunda variable mediadora se evaluó mediante la pregunta "¿Le ha faltado dinero para cubrir sus necesidades?", las opciones de respuesta se dicotomizaron en: necesidades satisfechas (respuestas "nunca" y "casi nunca") e insatisfechas ("algunas veces", "muchas veces", "casi siempre" y "siempre"). El distrés piscológico se evaluó mediante las preguntas de salud mental del cuestionario SF36 ${ }^{23}$. Se asignó un valor a cada opción de respuesta (de 0 para nunca a 100 para siempre), siendo los valores más altos equivalentes a los niveles más altos de distrés psicológico. Para calcular el puntaje de distrés, se sumaron los puntajes de todas las respuestas y se dividieron entre el número de preguntas. El alfa de Cronbach de esta escala de distrés fue 0.86 , lo que evidencia su validez interna. Se indagó la existencia de siete síntomas relacionados con los efectos secundarios del TAR (dolor de cabeza, fatiga o debilidad, nauseas, molestias estomacales como vómito, diarrea o dolor de estómago; mareo, los medicamentos hacen que las cosas sepan mal o extraño y adormecimiento de manos o boca). Se estimó el promedio del puntaje asignado a las respuestas (0: nunca a 5: siempre) sobre síntomas para ello se sumaron los puntajes de cada pregunta y se dividió entre el número de preguntas.

Se estimaron las estadísticas descriptivas de las variables sociodemográficas de la población total y de acuerdo a los grupos de IA. Se comparó la frecuencia de no-adherencia entre los grupos de la variable independiente y se realizó el análisis bivariado usando la prueba de chi cuadrada para conocer si existían diferencias estadísticamente significativas $(p<0.050)$. Dado que no existieron diferencias en la no adherencia en el día previo, no se presentan los resultados en tablas. Se realizaron modelos de regresión logística para obtener la asociación entre la IA y la adherencia al tratamiento ajustada por otras variables. Estos análisis se realizaron con el programa SPSS versión 21.

Para evaluar si los mediadores postulados explicaban la asociación entre la IA y la adherencia al TAR se estimaron modelos de regresión logística. Un mediador debe cumplir con ciertas premisas $^{24}:$ a) el mediador debe ser más frecuente en el grupo expuesto (Tabla 1), b) el mediador debe estar asociado con los eventos de salud estudiados (adherencia al TAR) (Tabla 2) y c) al ser incorporado en los modelos de regresión debe resultar en disminución de las estimaciones de las asociaciones entre la exposición (IA) y el evento (adherencia al TAR). Para la tercera premisa se 
Tabla 1. Características sociodemográficas de acuerdo a la inseguridad alimentaria $(n=557)$.

\begin{tabular}{|c|c|c|c|c|c|c|c|c|c|}
\hline & \multirow{2}{*}{\multicolumn{2}{|c|}{ Total $^{\text {a }}$}} & \multicolumn{7}{|c|}{ Diferencias de acuerdo a inseguridad alimentaria ${ }^{\mathrm{b}}$} \\
\hline & & & \multicolumn{2}{|c|}{ SA } & \multicolumn{2}{|c|}{ IAL } & \multicolumn{2}{|c|}{ IAM } & \multirow[b]{2}{*}{$\mathbf{p}$} \\
\hline & $\mathbf{n}$ & M & $\mathbf{n}$ & $\mathbf{M}$ & $\mathbf{n}$ & M & $\mathbf{n}$ & $\mathbf{M}$ & \\
\hline \multirow[t]{2}{*}{ Edad (media) } & 557 & 36.6 & 232 & 37.0 & 142 & 34.7 & 183 & 37.6 & 0.073 \\
\hline & & $\%$ & & $\%$ & & $\%$ & & $\%$ & \\
\hline Total & & & 232 & 41.7 & 142 & 25.5 & 183 & 32.8 & \\
\hline \multicolumn{10}{|l|}{ Sexo } \\
\hline Hombres heterosexuales & 112 & 20.1 & 63 & 56.3 & 25 & 22.3 & 24 & 21.4 & 0.000 \\
\hline Hombres homo y bisexuales & 336 & 60.3 & 137 & 40.8 & 94 & 28.0 & 105 & 31.3 & \\
\hline Mujeres & 109 & 19.6 & 32 & 29.4 & 23 & 21.1 & 54 & 49.5 & \\
\hline \multicolumn{10}{|l|}{ Estado civil } \\
\hline Soltero & 347 & 62.3 & 151 & 43.5 & 91 & 26.2 & 105 & 30.3 & 0.267 \\
\hline Casado-unión libre & 146 & 26.2 & 57 & 39.0 & 39 & 26.7 & 50 & 34.2 & \\
\hline Divorciado-viudo & 64 & 11.5 & 24 & 37.5 & 12 & 18.8 & 28 & 43.8 & \\
\hline \multicolumn{10}{|l|}{ Escolaridad del jefe de familia } \\
\hline Primaria o menos & 74 & 13.3 & 18 & 24.3 & 12 & 16.2 & 44 & 59.5 & 0.000 \\
\hline Secundaria & 142 & 25.5 & 32 & 22.5 & 42 & 29.6 & 68 & 47.9 & \\
\hline Bachillerato o carrera técnica & 215 & 38.7 & 104 & 48.4 & 56 & 26.0 & 55 & 25.6 & \\
\hline Licenciatura y más & 125 & 22.5 & 77 & 61.6 & 32 & 25.6 & 16 & 12.8 & \\
\hline \multicolumn{10}{|l|}{ Institución } \\
\hline ISSSTE & 100 & 18.0 & 63 & 63.0 & 20 & 20.0 & 17 & 17.0 & 0.000 \\
\hline SS-GDF & 457 & 82.0 & 169 & 37.0 & 122 & 26.7 & 166 & 32.9 & \\
\hline
\end{tabular}

SA, seguridad alimentaria; IAL, inseguridad alimentaria leve; IAM, inseguridad alimentaria moderada; M, media. Se estimaron las pruebas de chi cuadrado (variables categóricas) y de ANOVA (variables continuas) para conocer si existían diferencias estadísticamente significativas entre grupos de inseguridad alimentaria. ${ }^{a}$ Los porcentajes suman $100 \%$ sobre las columnas. ${ }^{b}$ Los porcentajes suman $100 \%$ sobre las filas.

Tabla 2. Eventos y mediadores de acuerdo al grado de inseguridad alimentaria $(n=557)$.

\begin{tabular}{|c|c|c|c|c|c|c|c|c|c|}
\hline & \multirow{2}{*}{\multicolumn{2}{|c|}{ Total $^{\mathrm{a}}$}} & \multicolumn{7}{|c|}{ Diferencias de acuerdo a inseguridad alimentaria ${ }^{b}$} \\
\hline & & & \multicolumn{2}{|c|}{ SA } & \multicolumn{2}{|c|}{ IAL } & \multicolumn{2}{|c|}{ IAM } & \multirow[b]{2}{*}{$\mathbf{p}$} \\
\hline & n & $\%$ & $\mathbf{n}$ & $\%$ & $\mathbf{n}$ & $\%$ & $\mathbf{n}$ & $\%$ & \\
\hline \multicolumn{10}{|l|}{ Adherencia } \\
\hline Omisión de toma en semana previa & 68 & 12.2 & 26 & 11.2 & 12 & 8.5 & 30 & 16.4 & 0.079 \\
\hline Baja adherencia en semana previa & 29 & 5.2 & 11 & 4.7 & 2 & 1.4 & 16 & 8.7 & 0.012 \\
\hline Omisión de toma en mes previo & 131 & 23.3 & 49 & 21.1 & 22 & 15.5 & 60 & 32.8 & 0.001 \\
\hline Baja adherencia en mes previo & 33 & 5.9 & 8 & 3.4 & 4 & 2.8 & 21 & 11.5 & 0.001 \\
\hline \multicolumn{10}{|l|}{ Mediadores } \\
\hline Falta de tiempo & 129 & 23.2 & 25 & 10.8 & 36 & 25.4 & 68 & 37.2 & 0.000 \\
\hline \multirow[t]{2}{*}{ Necesidades insatisfechas } & 135 & 24.3 & 8 & 3.5 & 31 & 21.8 & 96 & 52.5 & 0.000 \\
\hline & $\mathbf{n}$ & $\mathbf{M}$ & $\mathbf{n}$ & $\mathbf{M}$ & $\mathbf{n}$ & $\mathbf{M}$ & $\mathbf{n}$ & $\mathbf{M}$ & \\
\hline Distrés psicológico & 557 & 34.5 & 232 & 29.3 & 142 & 36.0 & 183 & 39.9 & 0.000 \\
\hline Efectos secundarios & 557 & 1.01 & 232 & 0.85 & 142 & 0.98 & 183 & 1.23 & 0.001 \\
\hline
\end{tabular}


estimaron modelos de regresión utilizando el comando $k h b$ para STATA ${ }^{25}$. Este procedimiento proporciona estimaciones sin sesgo de las contribuciones de variables mediadoras tanto categóricas como continuas, además de que permite incluir variables confusoras de efecto. Con dicho comando se estimó en qué medida las diferencias en la adherencia al TAR de acuerdo a la IA eran explicadas por la falta de tiempo debido a actividades laborales y familiares y por el distrés psicológico (Tabla 3).

Tabla 3. Modelos de regresión logística teniendo como variables dependientes la omisión y baja adherencia en la toma de medicamentos y como variable independiente la inseguridad alimentaria.

\begin{tabular}{lcccc}
\hline & \multicolumn{2}{c}{$\begin{array}{c}\text { Inseguridad } \\
\text { leve }\end{array}$} & $\begin{array}{c}\text { Inseguridad } \\
\text { moderada }\end{array}$ \\
\cline { 2 - 5 } & $\mathbf{R M}$ & IC & RM & IC \\
\hline $\begin{array}{c}\text { Semana previa } \\
\text { Omisión de toma }\end{array}$ & 0.71 & $0.34-1.48$ & 1.52 & $0.82-2.84$ \\
$\begin{array}{c}\text { Baja adherencia } \\
\text { Mes previo }\end{array}$ & 0.27 & $0.59-1.26$ & 1.95 & $0.82-4.66$ \\
$\begin{array}{c}\text { Omisión de toma } \\
\text { Baja adherencia }\end{array}$ & 0.71 & $0.41-1.25$ & 2.10 & $1.29-3.43$ \\
\hline
\end{tabular}

RM, razón de momios; IC, intervalo de confianza al 95\%. Modelos ajustados por sexo, edad, estado civil, escolaridad del jefe de familia e institución. El grupo de referencia fueron las personas con seguridad alimentaria.

\section{Resultados}

En la Tabla 4 se muestran las características sociodemográficas de la población. La media de edad fue de 36.6 años. La mayor parte de la población eran hombres homosexuales y bisexuales. Casi tres cuartas partes reportaron ser solteros y $82.0 \%$ eran usuarios de la clínica de la SS-GDF. El 38.7\% vivían en hogares en donde el jefe de familia tenía el bachillerato como grado de máximo de escolaridad. Más de la mitad de la población experimentaba algún grado de IA. La mayoría de los hombres heterosexuales tenían SA y la mayoría de mujeres tenían IA leve o moderada. La mayoría de personas con escolaridad de primaria y secundaria presentaban IA tanto leve como moderada; al mismo tiempo la mayoría con bachillerato y licenciatura se situó en el grupo de SA.

La baja adherencia tanto en la semana como en el mes previo, así como la omisión en el mes previo, fue mayor en el grupo con IA moderada en comparación con el grupo de SA (Tabla 1). Casi una cuarta parte de los participantes tenía falta de tiempo para sí mismos debido a actividades laborales o familiares o no tenía suficiente dinero para cubrir sus necesidades. Ambas situaciones fueron más comunes entre las personas con IA. El puntaje promedio de distrés psicológico en la población total fue de 34.5 y en comparación con el grupo con SA fue mayor entre los grupos con IA. La puntuación media de efectos secundarios fue de 1.01 y fue mayor en los grupos de IA que en el de SA.

En los modelos de regresión logística (Tabla 5) se observó que en comparación con las perso-

Tabla 4. Diferencias en omisión de medicamento y baja adherencia en el mes previo de acuerdo a posibles variables mediadoras.

\begin{tabular}{|c|c|c|c|c|c|}
\hline & \multicolumn{3}{|c|}{ Falta de tiempo } & \multicolumn{2}{|c|}{ Distrés psicológico } \\
\hline & Sí & No & $\mathbf{p}$ & B & $\mathbf{p}$ \\
\hline & $\%$ & $\%$ & & & \\
\hline Omisión de toma & 30.2 & 21.5 & 0.040 & 0.012 & 0.013 \\
\hline \multirow[t]{2}{*}{ Baja adherencia } & 10.1 & 4.7 & 0.023 & 0.020 & 0.014 \\
\hline & \multicolumn{3}{|c|}{ Necesidades insatisfechas } & \multicolumn{2}{|c|}{ Efectos secundarios } \\
\hline Omisión de toma & 30.4 & 21.4 & 0.032 & 0.234 & 0.011 \\
\hline Baja adherencia & 11.9 & 4.0 & 0.001 & 0.541 & 0.000 \\
\hline
\end{tabular}

$\mathrm{B}$ = coeficiente de regresión logística en donde la variable independiente fueron el distrés psicológico y el puntaje de efectos secundarios; mientras que la variable dependiente fue la omisión o baja adherencia. 
Tabla 5. Modelos de regresión para evaluar la contribución de variables mediadoras. La variable independiente es la inseguridad alimentaria y las variables dependientes son la omisión de medicamentos y la baja adherencia.

\begin{tabular}{|c|c|c|c|c|}
\hline & \multicolumn{2}{|c|}{ Omisión en el mes previo } & \multicolumn{2}{|c|}{ Baja adherencia en el mes previo } \\
\hline & RM & IC95\% & RM & IC95\% \\
\hline \multicolumn{5}{|l|}{ Inseguridad alimentaria moderada } \\
\hline Modelo reducido $^{\text {a }}$ & 2.11 & 0.003 & 3.26 & 0.012 \\
\hline Modelo completo $^{\text {b }}$ & 1.70 & 0.062 & 2.17 & 0.147 \\
\hline Diferencia $^{c}$ & 1.24 & 0.107 & 1.50 & 0.071 \\
\hline Componentes de la diferencia ${ }^{\mathrm{d}}$ & & $\%$ & & $\%$ \\
\hline Falta de tiempo & & 6.7 & & 4.8 \\
\hline Necesidades insatisfechas & & 5.3 & & 12.5 \\
\hline Distrés psicológico & & 9.1 & & 2.0 \\
\hline Efectos secundarios & & 7.9 & & 14.9 \\
\hline
\end{tabular}

${ }^{a}$ RM, razón de momios. ${ }^{b}$ Es el mismo riesgo del modelo reducido, pero ajustado por las variables que se postulan funcionan como mediadoras, las cuales corresponden a aquellas con valores en componentes de la diferencia. Tanto en el modelo reducido como en el completo se ajustó por sexo, edad, estado civil, escolaridad del jefe de familia e institución. ${ }^{c}$ Diferencias entre el modelo completo y reducido. ${ }^{d}$ Es la contribución porcentual de cada variable a la diferencia entre modelos.

nas que tenían SA, las personas con IA moderada tuvieron mayor probabilidad de omisión en el mes previo y de baja adherencia.

La omisión de medicamentos y la baja adherencia durante el mes previo (Tabla 2) fue mayor entre las personas que tenían falta de tiempo en comparación con quienes no tenían falta de tiempo. Entre quienes habían tenido falta de dinero para cubrir sus necesidades hubo una mayor omisión y baja adherencia en comparación con quienes no habían tenido esa condición. De igual forma, la probabilidad tanto de omisión como de baja adherencia aumentó conforme incrementó el puntaje de distrés psicológico y conforme aumentó el puntaje de efectos secundarios agudos.

En la Tabla 3 se presentan los resultados del análisis de mediación. El hecho de que, en comparación con las personas que tienen SA, las que tienen IA moderada presenten mayor omisión de medicamentos durante el mes previo puede atribuirse en $9.1 \%$ a que tienen mayores niveles de distrés psicológico, en $7.9 \%$ a que tienen más efectos secundarios agudos, en $6.7 \%$ a la falta de tiempo para sí mismos y en $5.3 \%$ a las necesidades insatisfechas. En el caso de la baja adherencia, los efectos secundarios explicaron el 14.9\% de las diferencias, las necesidades insatisfechas el $12.5 \%$, la falta de tiempo el $4.8 \%$ y el distrés el $2.0 \%$.

\section{Discusión}

El principal hallazgo de este trabajo es que la IA moderada, pero no la leve, se asoció negativamente con la adherencia al TAR, medida ésta como la omisión de medicamentos o baja adherencia. Además, los cuatro mediadores postulados (necesidades insatisfechas, distrés psicológico, efectos secundarios y falta de tiempo) explicaron parte de las diferencias en adherencia de acuerdo al grado de IA.

En México, alrededor de la mitad de los hogares experimentan algún grado de $\mathrm{IA}^{4}$. Dicha proporción fue mayor (alrededor del 60\%) en las personas con VIH que participaron en el presente estudio. Parte de la diferencia se puede deber a que se incluyó una clínica que atiende a personas sin empleo formal y con menor ingreso al del resto de la población. Estas estadísticas muestran que la IA es un fenómeno frecuente en la ciudad de México, lo cual probablemente también se observe en otras ciudades de países de ingreso medio.

En las personas con VIH de la ciudad de México la IA se relaciona con dos o tres veces mayor probabilidad de no adherencia al TAR. Este hallazgo es congruente con lo observado en estudios epidemiológicos realizados con poblaciones 
en países de alto $^{7,8}$ y bajo ingreso ${ }^{14,15}$, así como con lo reportado en investigaciones cualitativas $^{9-12}$. Lo anterior implica que la IA puede representar una barrera importante para que las personas con VIH se adhieran al TAR, con lo cual se reducen los efectos benéficos de este último tanto para la persona con VIH (e.g. evitar la progresión de la infección ${ }^{1}$ e incrementar la sobrevivencia ${ }^{26}$ ) como a la salud pública (reducción de la transmisión $\left.{ }^{3}\right)$.

En estudios cualitativos realizados en países africanos $^{9,10,12}$ se hizo evidente que la sensación de hambre asociada con la IA acentuó los efectos secundarios del TAR (e.g. mareo o dolor de cabeza o estómago), lo cual puede redundar en menor adherencia al tratamiento Los resultados del presente estudio son acordes a esta observación, pues los efectos secundarios del TAR explicaron parte de las diferencias entre personas con y sin IA respecto a la adherencia al TAR.

En personas con VIH de la ciudad de México el distrés psicológico fue la variable que más explicó la relación entre IA y la omisión de medicamentos en el mes previo. En adultos la IA se asocia con un deficiente estado de salud mental, incluyendo el cumplimiento de los criterios diagnósticos de tamizaje para depresión mayor ${ }^{19,20}$. A su vez, un pobre estado de salud mental ha sido asociado con la no-adherencia al TAR ${ }^{21,27}$. Es decir, la salud mental deficiente (i.e. distrés psicológico) es otro potencial mecanismo por el cual la IA se relaciona con la no adherencia al TAR.

Otro mecanismo consiste en que las personas con IA tienen que destinar más tiempo para trabajar y conseguir recursos lo cual puede provocar que tengan fatiga física y mental ${ }^{12,18}$. Esta situación, a su vez, puede reducir el tiempo y capacidad para el autocuidado. En las personas con VIH de la ciudad de México la falta de tiempo debido a obligaciones familiares y laborales también explicó parte de la relación de la IA con la omisión de tomas y la baja adherencia. Personas con VIH de Uganda ${ }^{12}$ también mencionaron que debido a sus largas jornadas laborales olvidaban tomar sus medicamentos.

La experiencia de IA puede reducir la adherencia puesto que las personas pueden dar prioridad a la adquisición de alimentos sobre los gastos relacionados con el tratamiento del VIH (5) ya que la alimentación es una necesidad impostergable de satisfacer. En el presente estudio la insatisfacción de necesidad también medió la relación de la IA con la baja adherencia al TAR.
Una de las limitaciones de este trabajo es que se estudió a una muestra por conveniencia, por lo que su representatividad es limitada. Sin embargo, se incluyó a una institución que cubre a trabajadores de la económica formal y otra que está destinada a las personas sin seguridad social. Como cualquier estudio transversal, las asociaciones aquí reportadas no pueden ser consideradas como soporte para concluir que existen relaciones causales. Algunos de los otros instrumentos utilizados fueron desarrolladas para el estudio, por lo cual no se conoce su validez. Finalmente, las variables que se postularon mediadoras no explicaron el $65.8 \%$ de las diferencias en baja adherencia y el $71 \%$ de las diferencias en la omisión de tomas de las diferencias entre los grupos con SA e IA. Esto implica que otros mecanismos que no fueron estudiados pueden estar involucrados.

Es evidente que se requiere erradicar la IA, pues además de estar asociada con menor adherencia al TAR tiene efectos negativos en la salud y bienestar de las personas. La regulación de las condiciones de empleo y de los salarios son medidas gubernamentales que pueden tener impacto significativo en la reducción de la $\mathrm{IA}^{28}$. Los servicios de salud también pueden ser adecuados para reducir los efectos de la IA. En la historia clínica de las personas se puede indagar la existencia de IA. Mediante técnicas de consejería los profesionales de la salud pueden ayudar a las personas que experimentan IA a identificar estrategias para superar las barreras para la adherencia a la TAR que están relacionadas con la IA y que han sido identificadas en el presente estudio. A las personas con IA se les puede dar más facilidades para acceder al TAR como proveerles de medicamentos para periodos de tiempo más largos de lo usual (e.g. tres o cuatro meses) y darles seguimiento vía telefónica, de este modo requerirán ir con menos frecuencia a los servicios de salud, con lo cual ahorrarán tiempo y recursos.

México tiene una de las coberturas de TAR más altas $(85 \%)$ en América Latina y el mundo ${ }^{29}$. Sin embargo, el presente estudio muestra que a pesar de que las personas cuentan con el acceso al tratamiento, la IA puede limitar los beneficios de las políticas de acceso universal al TAR ya que puede estar asociada con menor adherencia a éste. Por tanto es indispensable la creación de políticas y programas encaminados a la erradicación de la IA o por lo menos la reducción de sus efectos negativos. 


\section{Colaboradores}

D Pérez-Salgado analizó los datos y elaboró el primer borrador del manuscrito. MS CompeanDardón diseñó el estudio, coordinó el trabajo de campo e hizo una revisión crítica del manuscrito. L Ortiz-Hernández diseñó el estudio, participó en el análisis de datos e hizo una revisión crítica del manuscrito.

\section{Agradecimientos}

Los autores agradecen a los participantes por su tiempo y paciencia para participar en el estudio. También agradecemos a los trabajadores y autoridades del Hospital Regional Lic. Adolfo López Mateos (Instituto de Seguridad y Servicios Sociales de los Trabajadores del Estado) y la Clínica Especializada Condesa (Secretaria de Salud del Distrito Federal) por permitirnos realizar el estudio. El proyecto recibió financiamiento del Programa de Mejoramiento del Profesorado (PROMEP) de la Secretaría de Educación Pública.

\section{Referências}

1. Bangsberg DR, Perry S, Charlebois ED, Clark RA, Roberston M, Zolopa AR, Moss A. Non-adherence to highly active antiretroviral therapy predicts progression to AIDS. AIDS 2001; 15(9):1181-1183.

2. Mayer KH, Wheeler DP, Bekker LG, Grinsztejn B, Remien RH, Sandfort TG, Beyrer C. Overcoming biological, behavioral, and structural vulnerabilities: new directions in research to decrease HIV transmission in men who have sex with men. J Acquir Immune Defic Syndr 2013; 63(Supl. 2):S161-S167.

3. Montaner JS, Lima VD, Barrios R, Yip B, Wood E, Kerr T, Shannon K, Harrigan PR, Hogg RS, Daly P, Kendall P. Association of highly active antiretroviral therapy coverage, population viral load, and yearly new HIV diagnoses in British Columbia, Canada: a population-based study. Lancet 2010; 376(9740):532-539.

4. Valencia-Valero RG, Ortiz-Hernández L. Disponibilidad de alimentos en los hogares mexicanos de acuerdo con el grado de inseguridad alimentaria. Salud Publica Mex 2014; 56(2):154-164.

5. Young S, Wheeler AC, McCoy SI, Weiser SD. A review of the role of food insecurity in adherence to care and treatment among adult and pediatric populations living with HIV and AIDS. AIDS Behav 2014; 18(Supl. 5):S505-S515.

6. Anema A, Kerr T, Milloy MJ, Feng C, Montaner JS, Wood E. Relationship between hunger, adherence to antiretroviral therapy and plasma HIV RNA suppression among HIV-positive illicit drug users in a Canadian setting. AIDS Care 2014; 26(4):459-465.

7. Kalichman SC, Cherry C, Amaral C, White D, Kalichman MO, Pope H, Swetsze C, Jones M, Macy R. Health and treatment implications of food insufficiency among people living with HIV/AIDS, Atlanta, Georgia. J Urban Health 2010; 87(4):631-641.

8. Peretti-Watel P, Spire B, Schiltz MA, Bouhnik AD, Heard I, Lert F, Obadia Y; VESPA Group. Vulnerability, unsafe sex and non-adherence to HAART: evidence from a large sample of French HIV/AIDS outpatients. Soc Sci Med 2006; 62(10):2420-2433.

9. Nagata JM, Magerenge RO, Young SL, Oguta JO, Weiser $\mathrm{SD}$, Cohen CR. Social determinants, lived experiences, and consequences of household food insecurity among persons living with HIV/AIDS on the shore of Lake Victoria, Kenya. AIDS Care 2012; 24(6):728-736.

10. Coetzee B, Kagee A, Vermeulen N. Structural barriers to adherence to antiretroviral therapy in a resource-constrained setting: the perspectives of health care providers. AIDS Care 2011; 23(2):146-151.

11. Goudge J, Ngoma B. Exploring antiretroviral treatment adherence in an urban setting in South Africa. J Public Health Pol 2011; 32(Supl. 1):S52-S64.

12. Weiser SD, Tuller DM, Frongillo EA, Senkungu J, Mukiibi N, Bangsberg DR. Food insecurity as a barrier to sustained antiretroviral therapy adherence in Uganda. Plos One 2010; 5(4):e10340. 
13. Au JT, Kayitenkore K, Shutes E, Karita E, Peters PJ, Tichacek A, Allen SA. Access to adequate nutrition is a major potential obstacle to antiretroviral adherence among HIV-infected individuals in Rwanda. AIDS 2006; 20(16):2116-2118.

14. Franke MF, Murray MB, Munoz M, Hernandez-Diaz S, Sebastian JL, Atwood S, Caldas A, Bayona J, Shin SS. Food insufficiency is a risk factor for suboptimal antiretroviral therapy adherence among HIV-infected adults in urban Peru. AIDS Behav 2011; 15(7):1483-1489.

15. Birbeck GL, Kvalsund MP, Byers PA, Bradbury R, Mang'ombe C, Organek N, Kaile T, Sinyama AM, Sinyangwe SS, Malama K, Malama C. Neuropsychiatric and socioeconomic status impact antiretroviral adherence and mortality in rural Zambia. Am J Trop Med Hygiene 2011; 85(4):782-789.

16. World Values Survey. Online Data Analysis: World Values Survey; [acessado 2015 jan 12]. Disponível em: http://www.worldvaluessurvey.org/WVSOnline.jsp.

17. Organización Panamericana de la Salud (OPAS), Organización Mundial de la Salud (OMS). América Latina y el Caribe avanzan hacia el acceso universal del tratamiento de VIH. Washington: OPAS, OMS; 2013. [acessado 2014 abr 1]. Disponível em: http://www.paho.org/uru/index.php?option=com_content\&view=article\&id=783:america-latina -y-el-caribe-avanzan-hacia-el-acceso-universal-del-tratamiento-de-vih \&catid=681:vih/sida\& Itemid $=239$.

18. Hernández ME, Pérez SD, Ortiz-Hernández L. Consecuencias alimentarias y nutricionales de la inseguridad alimentaria: la perspectiva de madres solteras. Rev Chil Nutr 2013; 40(4):351-356.

19. Hadley C, Patil CL. Food insecurity in rural Tanzania is associated with maternal anxiety and depression. Am J Human Biol 2006; 18(3):359-368.

20. Siefert K, Heflin CM, Corcoran ME, Williams DR. Food insufficiency and physical and mental health in a longitudinal survey of welfare recipients. $J$ Health Soc Behav 2004; 45(2):171-186.

21. Gordillo V, del Amo J, Soriano V, Gonzalez-Lahoz J. Sociodemographic and psychological variables influencing adherence to antiretroviral therapy. AIDS 1999; 13(13):1763-1769.

22. Comité Científico de la ELCSA. Escala Latinoamericana de Seguridad Alimentaria (ELCSA): manual de uso y aplicaciones. Santiago de Chile: Organización de las Naciones Unidas para la Agricultura y la Alimentación; 2012.

23. Zuniga MA, Carrillo-Jimenez GT, Fos PJ, Gandek B, Medina-Moreno MR. Evaluación del estado de salud con la Encuesta SF-36: resultados preliminares en México. Salud Publica Mex 1999; 41(2):110-118.
24. Baron RM, Kenny DA. The moderator-mediator variable distinction in social psychological research: conceptual, strategic, and statistical considerations. J Personality Soc Psychol 1986; 51(6):1173-1182.

25. Karlson KB, Holm A, Breen R. Comparing regression coefficients between Models using logit and probit: A new method. Washington: Working Paper Series Centre for Strategic Research in Education; 2010.

26. Wood E, Hogg RS, Yip B, Harrigan PR, O’Shaughnessy MV, Montaner JS. Effect of medication adherence on survival of HIV-infected adults who start highly active antiretroviral therapy when the CD4+ cell count is 0.200 to $0.350 \times 10$ (9) cells/L. Ann Internal Med 2003; 139(10):810-816.

27. Starace F, Ammassari A, Trotta MP, Murri R, De Longis P, Izzo C. Depression is a risk factor for suboptimal adherence to highly active antiretroviral therapy. J Acquir Immune Defic Syndr 2002; 31(Supl. 3):S136-139.

28. Comisión sobre Determinantes Sociales de la Salud. Subsanar las Desigualdades en una Generación. Alcanzar la equidad sanitaria actuando sobre los determinantes sociales de la salud. Geneva: Organización Mundial de la Salud; 2008.

29. Centro Nacional para la Prevención y el Control del VIH/SIDA (Censida). VIH/SIDA en México 2012. México: Censida; 2012.

Artigo apresentado em 25/04/2016

Aprovado em 20/06/2016

Versão final apresentada em 22/06/2016 
